\title{
BMJ Open Conceptualising sustainability in the surgical work of non-governmental organisations in low and middle-income countries : a scoping review protocol
}

\author{
Emma Goettke (D) , ${ }^{1}$ Clare Coultas (D) , ${ }^{2}$ Michelle White (1) , ${ }^{3}$ \\ Andrew J M Leather (1) ${ }^{1}$
}

To cite: Goettke E, Coultas C, White $\mathrm{M}$, et al. Conceptualising sustainability in the surgical work of non-governmental organisations in low and middleincome countries : a scoping review protocol. BMJ Open 2021;11:e048046. doi:10.1136/ bmjopen-2020-048046

- Prepublication history for this paper is available online. To view these files, please visit the journal online (http://dx.doi org/10.1136/bmjopen-2020048046).

Received 17 December 2020 Accepted 09 November 2021

D) Check for updates

C Author(s) (or their employer(s)) 2021. Re-use permitted under CC BY-NC. No commercial re-use. See rights and permissions. Published by BMJ.

${ }^{1}$ Population Health Sciences, King's College London Faculty of Life Sciences and Medicine, London, UK

${ }^{2}$ Institute of Psychiatry, Psychology \& Neuroscience, King's College London, London, UK

${ }^{3}$ Anaesthesia, Great Ormond Street Hospital for Children NHS Foundation Trust, London, UK

Correspondence to

Emma Goettke;

emma.1.goettke@kcl.ac.uk

\section{ABSTRACT}

Introduction Sustainability remains poorly defined in global surgery, yet is, nevertheless, crucial to the work of non-governmental organisations (NGOs) in low- and middle-income countries (LMICS) aimed at strengthening access to, and quality of, surgical and anaesthesia care. The objective of this protocol is to outline a scoping review that maps what is known in the literature about sustainability in NGO surgical work in LMICs.

Methods The application of Arksey and O'Malley's sixstage methodological framework is described: identifying research questions; identifying relevant publications; selecting publications; charting the data; reporting results; and stakeholder consultation. The review will include all study designs, as well as editorials, commentaries, sources of unpublished studies and grey literature. Three electronic databases will be searched. Two reviewers will use predefined and iteratively refined selection criteria based on the 'Population-Concept-Context' framework to independently screen titles and abstracts of citations from the search. Disagreements will be resolved together by the reviewers. Full-text screening will also be carried out independently by two reviewers. Disagreements at this stage will be resolved with a third party. The search strategy for grey literature will include searching in ProQuest Dissertations and Theses and the websites listed in a surgical NGO database. Further relevant citations will be identified by screening the reference lists of the included papers.

Ethics and dissemination This review will undertake a secondary analysis of data already collected and does not require ethical approval. The results will be disseminated through journals and conferences targeting surgical NGO stakeholders and global health academics.

\section{INTRODUCTION}

Surgical disease constitutes a significant portion of the global burden of disease, with an estimated $32.9 \%$ of all deaths in 2010 due to conditions requiring surgical care. ${ }^{1}$ According to the Lancet Commission on Global Surgery, 5 billion people are without access to safe, affordable and timely surgical care, and 9 in 10 people in low-income and
Strengths and limitations of this study

- This protocol is a timely presentation of a relevant scoping review question. Sustainability is not defined in the surgical work of non-governmental organisations (NGOs) and, as such, has not been the subject of review.

- The outlined scoping review approach allows for mapping the concept of sustainability and how it is understood and applied by NGOs regarding their work in LMICs.

- The proposed review describes a literature search conducted in English, possibly excluding publications about NGO surgical work in other languages.

- The search strategy uses the term 'surgical NGO.' However, the search strategy is sufficiently broad to capture papers that describe the surgical work of NGOs, even when such organisations are not primarily defined as surgical NGOs.

- Sustainability with respect to the surgical work of NGOs is probably best captured in internal reports and discussions. The authors have aimed to capture this through analysis of surgical NGO websites and in consultation with key stakeholders in stage 6 of the scoping review process.

lower middle-income countries cannot access basic surgical care. ${ }^{2}$ The commission also found that the poorest third of the world's population receives only $6.3 \%$ of the surgical procedures performed globally each year and that 143 million extra surgical procedures are required annually to meet the need, additional to the 313 million procedures currently performed per year. ${ }^{2}$ In January 2014, Jim Kim, former President of the World Bank, stated that: 'surgery is an indivisible, indispensable part of health care' and 'can help millions of people lead healthier, more productive lives'. ${ }^{2}$ The Lancet Commission set a vision of universal access to safe, affordable surgical and anaesthesia care when needed. ${ }^{2}$ The benefit to governments for 


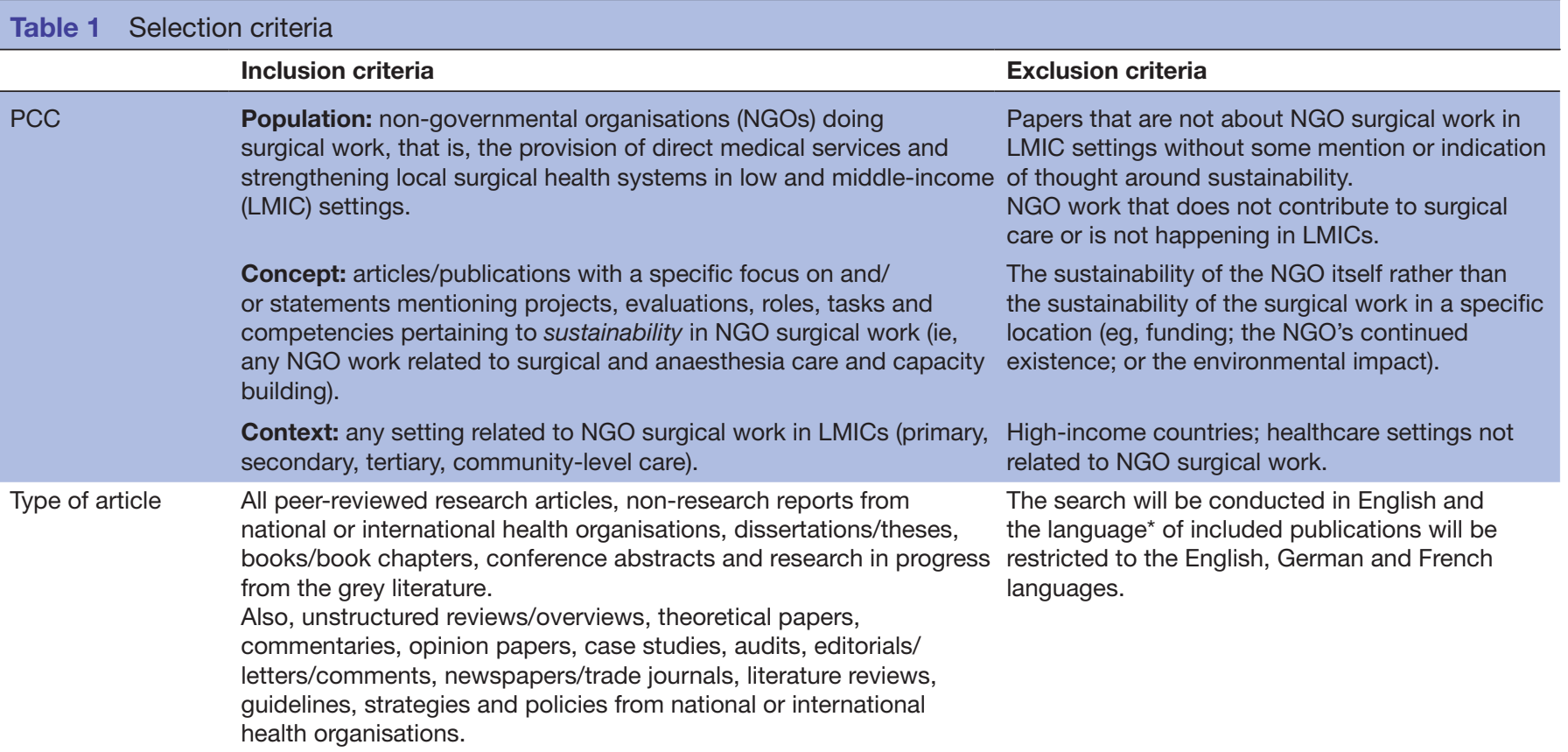

*Google Translate will be used on articles in other languages for the title and abstract stage. This will allow us to be aware of sustainability in the wider literature (see below). Interesting results that come up in other languages will be noted, but not included in our data extraction. PCC, Population-Concept-Context.

investing in sustainable surgical care is evident in the third edition of the Disease Control Priorities, which estimates that basic surgical care would avert 77.2 million disabilityadjusted life-years and 1.4 million deaths. ${ }^{4}$ As stated in the commission, "broad scale-up of quality surgical services will prevent deaths, limit disability, palliate suffering, promote economic growth, and help achieve maximum gains in health, welfare, and development for all." ${ }^{2}$ Yet, how sustainability is conceptualised in global surgery remains poorly defined. ${ }^{5}$

In 2015, the United Nations (UN) published the Sustainable Development Goals (SDGs), a series of 17 core goals, pertaining to different aspects of sustainability. The SDGs define sustainability broadly, covering aspects ranging from environmental sustainability to economic growth and provision of safe equitable healthcare. ${ }^{6}$ A subsequent Lancet Global Health Commission led by Kruk et al focused on health systems in the era of the SDGs, calling for an increased focus on delivering

\begin{tabular}{|c|c|c|}
\hline & Arksey and O'Malley framework' (p.22/-23) & $\begin{array}{l}\text { Enhancements proposed by Levac, } \\
\text { Colquhoun and O'Brien. }{ }^{12}(p .4-8)\end{array}$ \\
\hline 1. & Identifying the research question & $\begin{array}{l}\text { Clarifying and linking the purpose and } \\
\text { research question }\end{array}$ \\
\hline 2. & Identifying relevant studies & $\begin{array}{l}\text { Balancing feasibility with breadth and } \\
\text { comprehensiveness of the scoping } \\
\text { process }\end{array}$ \\
\hline 3. & Study selection & $\begin{array}{l}\text { Using an iterative team approach to } \\
\text { selecting studies and extracting data }\end{array}$ \\
\hline 4. & Charting the data & $\begin{array}{l}\text { Incorporating a numerical summary and } \\
\text { qualitative thematic analysis }\end{array}$ \\
\hline 5. & $\begin{array}{l}\text { Collating, summarizing and reporting the } \\
\text { results }\end{array}$ & $\begin{array}{l}\text { Identifying the implications of the study } \\
\text { findings for policy, practice or research }\end{array}$ \\
\hline 6. & Consultation (optional) & $\begin{array}{l}\text { Adopting consultation as a required } \\
\text { component of scoping study methodology }\end{array}$ \\
\hline
\end{tabular}

Figure 1 Six stages of a scoping review. ${ }^{21}$ sustainable high-quality care. ${ }^{7}$ The commission report underlined how global health targets have been skewed towards ensuring access to care, neglecting to simultaneously monitor and improve quality of care; a crucial oversight in that poor-quality care is estimated to account for $60 \%$ of avoidable deaths from conditions amenable to healthcare and lead to significant other adverse outcomes. $^{7}$ Consequently, it is argued that both quality and accessibility are necessary to ensure good sustainable healthcare in low and middle-income countries (LMICs).

In thinking about quality and access to surgical care in LMICs, one must consider the surgical work of nongovernmental organisations (NGOs), an important stakeholder group in the emerging global surgery community. In 2016, Ng-Kamstra et almade a first attempt at cataloguing surgical NGOs in existence. ${ }^{8}$ A surgical NGO is 'a not-forprofit group, principally independent from government, which is organized on a local, national, or international level to address issues in support of a public good', which specifically provides and/or strengthens surgical care in one or more LMICs. ${ }^{8}$ Shrime et al produced a framework for categorising these organisations into types based on how surgical services are provided: long-term specialty surgical hospitals and temporary surgical platforms, further subdivided into short-term surgical trips and selfcontained surgical platforms. ${ }^{5}$ Their review of the wide range of surgical NGOs practising worldwide used four dimensions of impact: effectiveness, cost-effectiveness, sustainability and role training. ${ }^{5}$ The authors show that the literature on the sustainability of NGO surgical work in LMICs is scarce and that the concept of sustainability in this context is poorly defined. ${ }^{5}$ 


\section{Box 1 Search terms}

Surgical non-governmental organisations
surgical NGO
s-NGO
surgical charity
surgical development work
international surgical NGO
international surgical non-governmental organisation
sustainability
sustainable
sustainably
long-term impact
legacy
Low- and Middle-Income Countries
low- or middle-income country
LMIC

Afghanistan; Albania; Algeria; Angola; Antigua and Barbuda; Argentina; Armenia;Azerbaijan; Bangladesh; Belarus; Belize; Benin; Bhutan; Bolivia; Bosnia and Herzegovina; Botswana; Brazil; Burkina Faso; Burundi; Cabo Verde; Cambodia; Cameroon; Central African Republic; Chad; People's Republic of China; Colombia; Comoros; Democratic Republic of Congo; Congo; Cook Islands; Costa Rica; Côte d'Ivoire; Cuba; Djibouti; Dominica; Dominican Republic; Ecuador; Egypt; El Salvador; Equatorial Guinea; Eritrea; Ethiopia; Fiji; Gabon; Gambia; Georgia; Ghana; Grenada; Guatemala; Guinea; Guinea-Bissau; Guyana; Haiti; Honduras; India; Indonesia; Iran; Iraq; Jamaica; Jordan; Kazakhstan; Kenya; Kiribati; Democratic People's Republic of Korea; Kosovo; Kyrgyzstan; Lao People's Democratic Republic; Lebanon; Lesotho; Liberia; Libya; Former Yugoslav Republic of Macedonia; Madagascar; Malawi; Malaysia; Maldives; Mali; Marshall Islands; Mauritania; Mauritius; Mexico; Micronesia; Moldova; Mongolia; Montenegro; Montserrat; Morocco; Mozambique; Myanmar; Namibia; Nauru; Nepal; Nicaragua; Niger; Nigeria; Niue; Pakistan; Palau; Palestine; Panama; Papua New Guinea; Paraguay; Peru; Philippines; Rwanda; Saint Helena; Samoa; São Tomé and Príncipe; Senegal; Serbia; Sierra Leone; Solomon Islands; Somalia; South Africa; South Sudan; Sri Lanka; Saint Lucia; Saint Vincent and the Grenadines; Sudan; Suriname; Swaziland; Syrian Arab Republic; Tajikistan; Tanzania; Thailand; TimorLeste; Togo; Tokelau; Tonga; Tunisia; Turkey; Turkmenistan; Tuvalu; Uganda; Ukraine; Uzbekistan; Vanuatu; Venezuela; Vietnam; Wallis and Futuna; West Bank and Gaza Strip; Yemen; Zambia; Zimbabwe

Mapping 'sustainability' in the surgical work of NGOs will contribute to a clearer understanding of the scope of the concept. Clarity about sustainability as a concept in global surgery is crucial as NGOs seek to align their aims to strengthen access to, and quality of, surgical and anaesthesia care in LMICs with the SDGs, where the sustainability of development work in the heath sector is important. The objective of the scoping review described in this protocol is to investigate and map the literature on sustainability in NGO surgical work. As a reference point, we will use the definition outlined in the 1987 Brundtland Commission Report, which describes sustainability on p 37 as meeting "the needs of the present without compromising the ability of future generations to meet their own needs." ${ }^{9}$ The given definition comprehends two key concepts, that of essential needs and that of limitations for the sake of being able to meet present and future needs. The UN expanded on their definition outlining four dimensions (society, environment, culture and economy) of the sustainability paradigm for thinking about the future in which environmental, societal and economic considerations are balanced in the pursuit of an improved quality of life." ${ }^{10}$ We hope to refine this understanding, mapping the way the term is used and contributing to a more nuanced definition, specific to the surgical work of NGOs. We use the World Bank Global Index from 2020 to define the LMICs we focus on. ${ }^{11}$

\section{METHODS AND ANALYSIS}

This review seeks to explore the concept of 'sustainability' in the literature of NGO surgical work in LMICs. Doing a scoping review is the most suitable approach for tackling an exploratory review of a multifaceted concept such as sustainability and related sustainability research questions. ${ }^{12}$ Unlike a systematic literature review which aims to answer specific questions, a scoping review maps the relevant literature, defines key terms and identifies research gaps to produce a broad overview of the field in question. ${ }^{12}$ In addition to searching databases with peerreviewed articles, it is important that the grey literature is searched as well. What is done by NGOs to enhance sustainable practice in surgery may not be connected to academic research and might not even be described using the term 'sustainability'. We will search the grey literature to ensure that practical applications of the concept of sustainability, which may remain unrecognised by academia, are captured. ${ }^{13}$ We base this protocol on the Preferred Reporting Items for Systematic Review and Meta-Analysis Protocols (PRISMA-P). ${ }^{14}$

The scoping review will use Arksey and O'Malley's methodological framework, with amendments to this framework proposed by Levac et $a l^{15}$ and by the Joanna Briggs Institute (JBI) (figure 1). ${ }^{1516}$ The general Arksey and O'Malley framework is made up of six stages: (1) identifying the research question; (2) identifying relevant studies; (3) study selection; (4) charting the data; (5) collating, summarising and reporting results; and (6) consultation.

\section{Stage 1: identifying the research question}

We broadly understand the concept of sustainability to refer to the positive and continued impact and/or legacy of NGO surgical work, that is, work possessing the "quality of being able to continue over a period of time'. ${ }^{17}$ We use the term sustainability loosely to refer to the short-term, mid-term and long-term impacts of NGO surgical work in LMICs, whether explicitly or implicitly referenced. NGO surgical work is defined as development work that aims to strengthen access to, and quality of, surgical and anaesthesia care. The definition of sustainability will be refined during the review.

As we become increasingly versed in the relevant literature, we will continue to develop the research questions iteratively and with the aim of taking a wide approach to help ensure that our search will generate breadth of 


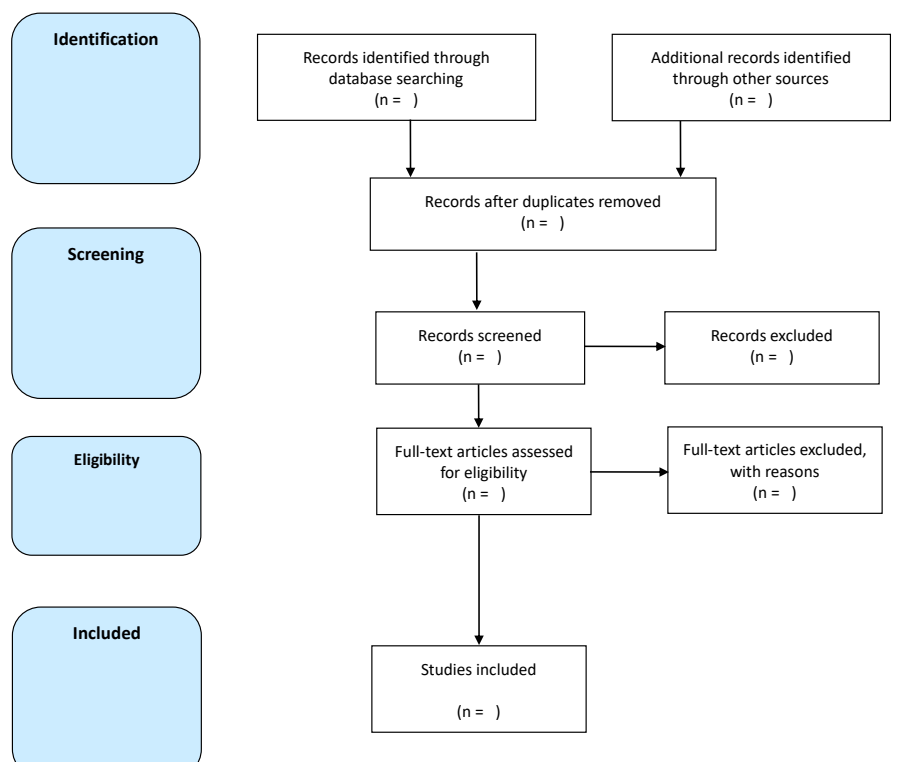

Figure 2 Preferred Reporting Items for Systematic Reviews and Meta-Analyses (PRISMA) flow chart. ${ }^{22}$

coverage. ${ }^{12}$ The objective is to determine what is known in the literature about sustainability in NGO surgical work in LMICs. Early forays into the literature were guided by a main question with several subquestions:

How is (the concept of) sustainability defined, understood, applied in programmatic work, and evaluated by NGOs regarding their surgical work in LMICs?

\begin{tabular}{|c|c|}
\hline $\begin{array}{l}\text { Citation } \\
\text { characteristics }\end{array}$ & Columns for extracted data \\
\hline $\begin{array}{l}\text { Details of the } \\
\text { publication }\end{array}$ & For example, author, title, source, year \\
\hline Surgical NGO & $\begin{array}{l}\text { For example, type (Shrime's categories }{ }^{5} \text { ), } \\
\text { country of origin, country location of work }\end{array}$ \\
\hline Research & $\begin{array}{l}\text { For example, aims/purpose of the study, } \\
\text { study design }\end{array}$ \\
\hline Sustainability & $\begin{array}{l}\text { For example, definition of sustainability/ } \\
\text { positive sustainable impact, framework for } \\
\text { evaluation of sustainable impact }\end{array}$ \\
\hline $\begin{array}{l}\text { (NGO) programmatic } \\
\text { work }\end{array}$ & $\begin{array}{l}\text { For example, theory of change (yes/no), logic } \\
\text { model (eg, inputs, outputs, outcomes) }\end{array}$ \\
\hline Intervention & $\begin{array}{l}\text { For example, design (type of intervention, } \\
\text { target population, etc), implementation } \\
\text { (context, duration, delivery methods, etc) }\end{array}$ \\
\hline $\begin{array}{l}\text { Monitoring and } \\
\text { evaluation }\end{array}$ & $\begin{array}{l}\text { For example, evaluation framework and } \\
\text { methodology (eg, implementation outcomes; } \\
\text { measures for capturing sustainability/impact) }\end{array}$ \\
\hline $\begin{array}{l}\text { Challenges and } \\
\text { opportunities }\end{array}$ & \\
\hline
\end{tabular}

NGO, non-governmental organisation.
- What approaches are taken, or what frameworks are being applied by NGOs, to sustainably strengthen access to, and quality of, surgical and anaesthesia care?

- What opportunities/challenges arise in the process of NGOs carrying out those strategies?

- How do NGOs evaluate the sustainable impact of their surgical work?

\section{Stage 2: identifying relevant studies}

As recommended by the PRISMA-P guidance, ${ }^{14}$ the search strategy with its key inclusion criteria will be comprehensively and iteratively developed, based on the Population-Concept-Context framework developed by Arksey and $\mathrm{O}^{\prime}$ Malley $^{12}$ and JBI, where the population is the surgical work of NGOs, the concept is sustainability and the context is LMICs. ${ }^{12} 16$ Table 1 includes the selection criteria, which will be further refined in the early stages of the literature search.

\section{Developing and applying our search strategy}

In the first step, we have conducted a preliminary search of one online peer-reviewed database relevant to the topic (PubMed). In the second step, we noted potentially relevant words in the titles and abstracts of papers found. This allowed us to compile a list of terms that can be used to inform our search strategy (see box 1). In the third step, we will run the search strategy in the PubMed, Web of Science and Scopus databases, and follow the selection procedure described in stage 3 .

Interesting results in other languages will be identified using Google Translate. We will note them, but not include them in our data extraction. In the final step 
(after selection), the reference lists of included studies will be searched using keywords related to sustainability and NGO surgical work, as outlined in our search strategy, to identify any additional relevant literature. The same process will apply for grey literature databases. Our process of searching the grey literature and the surgical NGO websites is described in the next paragraph.

\section{Grey literature search strategy}

We anticipate that we might miss important ways in which sustainability is understood and applied if we limit ourselves to the peer-reviewed literature. ${ }^{13}$ We will therefore access the grey literature by searching in ProQuest Dissertations and Theses, Google, and the websites listed in Ng-Kamstra et als surgical NGO database. ${ }^{8}$ Our search strategies for ProQuest Dissertations and Theses will parallel the peer-reviewed database search strategy described above. When searching Google, we will not apply language filters and will include English, French, and German language results. Interesting results that come up in other languages will be noted, but not included in our data extraction. The first 200 results of the Google search will be reviewed, based on the titles and two to three lines of text for each result. ${ }^{18}$ The surgical NGO websites ${ }^{8}$ will be hand searched using the key search terms from the database search strategies.

\section{Stage 3: study selection}

Study selection will proceed in three steps for the academic databases and the grey literature sources, starting with selection based on title and abstract screening by two independent researchers. Disagreements in this step will be resolved by the two researchers together. The selected and agreed-upon citations will then be read in full by the two researchers. Disagreements at this point will be resolved by the researchers in consultation with a third party. Exclusion will be explained for texts not included after full-text perusal in this second step. In the third step, recommended by JBI, the reference lists of the included citations will be hand searched for relevant publications. We will use the program Zotero to store and keep track of our citations at the different stages (see figure 2). Zotero will also allow us to identify and remove duplicates.

\section{Stage 4: charting the data}

We have developed an a priori extracting form (see table 2 for a summarised version) for charting data from the academic and grey literature sources. The surgical NGO websites will be hand searched by one of the authors based on the extracting form. Additional categories may emerge during data extraction; additional columns will be added as necessary. Three independent researchers will work together to accomplish the data extraction.

\section{Stage 5: collating, summarising and reporting results}

All the included sources will be charted in the data charting form. The charted data will be analysed thematically. ${ }^{19}$ The tables will be how we will chart the results of the review; it will provide the basis for our discussion in the review and for the definition of sustainability in NGO surgical work in LMICs. We will be looking at different approaches taken, and ways sustainability is conceptualised and put into practice in different contexts. To help ensure our review is rigorous, we will use a checklist specifically developed for reporting scoping reviews-the Preferred Reporting Items for Systematic Reviews and Meta-Analyses: extension for Scoping Reviews. ${ }^{20}$

\section{Stage 6: consultation}

We will purposively sample one to two surgical NGOs from the surgical NGO database, based on their engagement and experience with the concept of sustainability. We will request to informally consult with them about our findings and include this in our discussion section.

\section{Patient and public involvement}

This protocol presents a plan for a review of previously published research. Patients and members of the public were therefore not involved in the development of this protocol.

\section{ETHICS AND DISSEMINATION}

The results will be disseminated through a peer-reviewed publication and at conferences targeting stakeholders, to whom sustainability in NGO surgical work is relevant. The results will also be used to inform further research, exploring sustainability in the medical capacity-building work of a surgical NGO (Mercy Ships) operating in subSaharan Africa. This scoping review does not require ethical approval.

\section{Twitter Michelle White @mcwdoc}

Contributors EG, CC, MW and AJML were involved in the manuscript in the following way: substantial contributions to the conception or design of the work; or the acquisition, analysis or interpretation of data for the work; and drafting the work or revising it critically for important intellectual content; and final approval of the version to be published; and agreement to be accountable for all aspects of the work in ensuring that questions related to the accuracy or integrity of any part of the work are appropriately investigated and resolved.

Funding EG is supported by funding from her employer, Mercy Ships, to do her $\mathrm{PhD}$. CC is supported by the National Institute for Health Research (NIHR) Applied Research Collaboration South London (NIHR ARC South London) at King's College Hospital NHS Foundation Trust. The views expressed are those of the author[s] and not necessarily those of the NIHR or the Department of Health and Social Care. AJML is supported by the NIHR Global Health Research Unit on Health System Strengthening in sub-Saharan Africa, King's College London (GHRU 16/136/54), and by the Antibiotic use across Surgical Pathways-Investigating, Redesigning and Evaluating Systems (ASPIRES) research programme in LMICs, funded by the Economic and Social Research Council.

Disclaimer The views expressed are those of the authors and not necessarily those of the NIHR or the Department of Health and Social Care.

Competing interests None declared.

Patient consent for publication Not required.

Provenance and peer review Not commissioned; externally peer reviewed.

Open access This is an open access article distributed in accordance with the Creative Commons Attribution Non Commercial (CC BY-NC 4.0) license, which permits others to distribute, remix, adapt, build upon this work non-commercially, and license their derivative works on different terms, provided the original work is properly cited, appropriate credit is given, any changes made indicated, and the use is non-commercial. See: http://creativecommons.org/licenses/by-nc/4.0/. 


\section{ORCID iDs}

Emma Goettke http://orcid.org/0000-0002-9576-9415

Clare Coultas http://orcid.org/0000-0002-8506-8287

Michelle White http://orcid.org/0000-0002-5118-1054

Andrew J M Leather http://orcid.org/0000-0003-0500-5962

\section{REFERENCES}

1 Shrime MG, Bickler SW, Alkire BC, et al. Global burden of surgical disease: an estimation from the provider perspective. Lancet Glob Health 2015;3 Suppl 2:S8-9.

2 Meara JG, Leather AJM, Hagander L, et al. Global surgery 2030: evidence and solutions for achieving health, welfare, and economic development. Lancet 2015;386:569-624.

$3 \mathrm{Kim}$ JY. Opening address to the inaugural "The Lancet Commission on Global Surgery" meeting - Google Scholar. World Bank, 2014. Available: https://scholar.google.com/ scholar?hl=en\&q=Kim+JY+Opening+address+to+the+ inaugural+"The+Lancet+Commission+on+Global+Surgery"+ meeting.+The+World+Bank.+Jan+17\%2C+2014.+Boston\%2C+ MA\%2C+USA.http\%3A\%2F\%2Fwww.globalsurgery.info\%2Fwpcontent\%2Fuploads\%2F2014\%2F0 [Accessed 25 Nov 2020].

4 Bickler SW, Weiser TG, Kassebaum N. Essential Surgery - Chapter 2: Global Burden of Surgical Conditions. 3 edn. World Bank Group, 2015.

5 Shrime MG, Sleemi A, Ravilla TD. Charitable platforms in global surgery: a systematic review of their effectiveness, costeffectiveness, sustainability, and role training. World J Surg 2015;39:10-20.

6 United Nations. Transforming our world: the 2030 agenda for sustainable development, 2015. Available: sustainabledevelopment. un.org

7 Kruk ME, Gage AD, Arsenault C, et al. High-Quality health systems in the sustainable development goals era: time for a revolution. Lancet Glob Health 2018;6:e1196-252.

8 Ng-Kamstra JS, Riesel JN, Arya S, et al. Surgical Non-governmental organizations: global surgery's unknown nonprofit sector. World $J$ Surg 2016;40:1823-41.
9 UN Secretary General. Our common future: report of the world Commission on environment and development $1987 \mathrm{https}: / /$ www.are.admin.ch/are/en/home/media/publications/sustainabledevelopment/brundtland-report.html

10 Sustainable Development. UNESCO. Available: https://en.unesco. org/themes/education-sustainable-development/what-is-esd/sd [Accessed 23 Jul 2021].

11 World Bank. World bank country and lending groups - world bank data help desk, 2020. Available: https://datahelpdesk.worldbank.org/ knowledgebase/articles/906519-world-bank-country-and-lendinggroups [Accessed 15 Dec 2020].

12 Arksey H, O'Malley L. Scoping studies: towards a methodological framework. Int J Soc Res Methodol 2005;8:19-32.

13 Mahood Q, Van Eerd D, Irvin E. Searching for grey literature for systematic reviews: challenges and benefits. Res Synth Methods 2014:5:221-34.

14 Shamseer L, Moher D, Clarke M, et al. Preferred reporting items for systematic review and meta-analysis protocols (PRISMA-P) 2015: elaboration and explanation. BMJ 2015;349:g7647.

15 Levac D, Colquhoun H, O'Brien KK. Scoping studies: advancing the methodology. Implement Sci 2010;5:69 http://www.cihr-irsc.ca

16 Aromataris E, Munn Z. Joanna Briggs Institute Reviewer's Manual, 2017. Available: https://reviewersmanual.joannabriggs.org/

17 Sustainability | meaning in the Cambridge English dictionary. Available: https://dictionary.cambridge.org/dictionary/english/ sustainability [Accessed 25 Nov 2020].

18 Godin K, Stapleton J, Kirkpatrick SI, et al. Applying systematic review search methods to the grey literature: a case study examining guidelines for school-based breakfast programs in Canada. Syst Rev 2015:4:138.

19 Green J, Thorogood N. Qualitative methods for health research. 3 edn. Sage Publications, 2014.

20 Tricco AC, Lillie E, Zarin W, et al. PRISMA extension for scoping reviews (PRISMA-ScR): checklist and explanation. Ann Intern Med 2018;169:467-73.

21 Peters MDJ, Godfrey C, Mclnerney P. Chapter 11: Scoping Reviews (2020 Version). In: Aromataris E, Munn Z, eds. The Joanna Briggs Institute Reviewers' Manual 2015: Methodology for JBI Scoping Reviews, 2020. https://reviewersmanual.joannabriggs.org/

22 Peters MDJ, Godfrey CM, Khalil H, et al. Guidance for conducting systematic scoping reviews. Int J Evid Based Healthc 2015;13:141-6. 